\title{
Analytical Solution to FRW Metric with Variable Speed of Light
}

\author{
Gabriel W. Joseph, Terkaa V. Targema* and M. O. Kanu \\ Department of Physics, Taraba State University, P. M. B. 1167, Jalingo, Taraba State, Nigeria \\ *Email:vicnelson002@gmail.com
}

\begin{abstract}
According to the principle of general covariance, the laws of physics are the same in all reference frames. The controversial theory of the Varying Speed of Light (VSL) contradicts the principle of general covariance. Fortunately the VLS theory explains some crucial issues in cosmology such as Lorentz variance, biometric theories, locally Lorentz variance, cosmological constant problem, horizon and flatness problems. Also, recent astronomical observations from quasar show that the fine structural constant depends on redshift and therefore, varies with cosmological time. In other to harness this fascinating and published knowledge, two models where used in this work. 1. Cosmology with variables c; here the Friedmann-Robertson-Walker (FRW) is used in the Einstein field equation with variable $\mathrm{c}$ and $\Lambda$ terms to obtain the scale factor, which shows the continuous exponential expansion of the universe. 2. Variation of the speed of light as a function of the scale factor of the universe; here we obtained: a good approximation to estimate the current age of the universe. The scale factor of the universe depends its content given by the equation of state parameter $\omega$. We obtained the deceleration parameter in terms of the Hubble parameter. We arrived at a conclusion that the universe was decelerating at $\omega=1$, accelerating at $\omega=1 / 3$ and the Hubble parameter diverges at the beginning and end of the universe.
\end{abstract}

Keywords: VSL, Hubble Parameter, Friedmann Equation, Equation of State Parameter

\section{INTRODUCTION}

The principle of general covariance asserts that the laws of Physics take the same form in all frames of reference. This form the bases in which Einstein's theory of General Relativity hold its ground. However, recent Astronomical observations from quasar linked the fine structure constant to depend on redshift - hence suggesting its variation with cosmological time [1] According to [2], observational data relating the luminosity-redshift from type Ia supernovae now at range $z \sim 1$. The varying speed of light theory is geared towards explaining: hard breaking the Lorentz invariance, bimetric theories (with postulates that the speeds of gravity and light are not the same), locally Lorentz invariance. By varying the speed of light, the principle of general covariance is violated and the laws of Physics are now valid only in some special frames. Varying Speed of Light (VSL) theory has successfully handled some fundamental contention in cosmology such as flatness, horizon and Lambda problem. [3] introduced the VSL scenario using the power law with $c=c_{0} a(t)^{n}$ in which $\mathrm{c}$ changes from $c^{-}$to $c^{+}$during the phase transition. Where $a(t)^{n}$ is the scale factor and $c_{0}$ is a constant. It is worth noting that changes in $\mathrm{c}$ do not influence the geometry of the Universe.

Aside $c$, other physical constants of nature $G$ and $\Lambda$ that occurred in both the Einstein field equation and Friedmann equation have been varied in the literatures. In the Einstein field equation for instance, the gravitational constant $G$ serves as a coupling constant between matter and gravity [4]. At the infant stage of our universe, G appears to be time dependent [5]. In other 
to unify gravitation and elementary particles Physics, appendages of Einstein's Gravitation with time dependent $G$ have been introduced [6]. By so doing, $\Lambda$ which is interpreted as due to quantum mechanics and vacuum Physics is also varied. The inconsistency between observed value of the vacuum energy density and the theoretical large value of zero point-point energy as envisaged by the quantum field theory is called cosmological constant problem. [7] gives an elaborate discussion of the catastrophe and the effects of cosmology with time varying physical constants.

According to [8], the variation of the cosmological constant with time takes the form $\Lambda(t)=$ $B t^{-2}$ while, [9] opines that $\Lambda \propto a^{-2}$ where $a(t)$ is the scale factor in the Robertson-Walker metric. In another model, [10], proposed that $\Lambda \propto \frac{\ddot{a}}{a}$. Working on the Robertson-Walker universe with variable $\Lambda$ and $\mathrm{G},[11]$ proposed that $G \rho \propto \frac{\Lambda}{8 \pi}$.

In this paper, we study a model involving a time depend speed of light in FRW metric, and study its cosmological dynamics, in order to address the different epochs during the evolution of the universe. The structure of this paper is as follows: In section II, we study FRW cosmology with variable speed of light. In section III, we obtain solution for the universe in which the speed of light varies as the rate of change of the scale factor and we conclude in section IV.

\section{Cosmology with Variable c}

For variable c, the spatially Homogeneous and isotropic Friedmann-Robertson-Walker (FRW) metric [12] becomes

$$
d s^{2}=-c(t)^{2} d t^{2}+a^{2}(t)\left[\frac{d r^{2}}{1-k r^{2}}+r^{2} d \theta^{2}+r^{2} \sin ^{2} \theta d \varphi^{2}\right]
$$

Where $c(t) \neq 1$ and $k=-1,0,+1$ remain the respective curvature parameter for the open, flat and closed universe. The Einstein field equation with variable $c$ and $\Lambda$ terms is given by

$$
R_{\mu v}-\frac{1}{2} g_{\mu v} R+\Lambda(t)=\frac{8 \pi G T_{\mu v}}{c^{4}}
$$

Assuming that the matter in the universe is represented by the energy-momentum tensor of a perfect fluid given

$$
T_{\mu v}=\left(\rho+\frac{p}{c^{2}}\right) u_{\mu} u_{v}-p g_{\mu v}
$$

Where $\rho$ and $p$ denote respective mass density and pressure of the matter and $u_{\mu}$ is the four velocity satisfying

$$
u_{\mu}=(0,0,0, c)
$$

The solution to the Einstein [13] become

$$
\begin{aligned}
& 3 H^{2}=8 \pi G \rho+\Lambda c^{2}-3 \frac{k c^{2}}{a^{2}} \\
& 2 \frac{\ddot{a}}{a}+\left(\frac{\dot{a}}{a}\right)^{2}+\frac{k c^{2}}{a^{2}}-\Lambda c^{2}=-\frac{8 \pi G P}{c^{2}}
\end{aligned}
$$

For flat space, $k=0$ and using the equation of state of the form

$$
p=(\omega-1) \rho c^{2}
$$

We obtain

$$
\frac{\ddot{a}}{a}+\left(\frac{3 \omega-2}{2}\right)\left(\frac{\dot{a}}{a}\right)^{2}-\omega \Lambda c^{2}=0
$$

According to [1], there is a variation between $c$ and $\Lambda$ and suggest that either $c$ to vary and $\Lambda$ to be constant and vice versa. For instance, $c \propto a^{-r}$ or $c \propto H^{u}$ and $\Lambda=$ constant corresponds to $\Lambda \propto$ $a^{-2 r}$ or $\Lambda \propto H^{2 u}$ and $\mathrm{c}=$ constant. This follows the variation law that asserts that, one can take 
either $\mathrm{c}$ or $\Lambda$ to be time depend provided on the parameters is held constant. It is obvious that equation (8) do not have analytical solution and [1] resolve to obtaining a numerical solution by setting $\Lambda=$ constant and $\omega=1$ and introducing the density parameters terms $\Omega_{m}=\frac{8 \pi G}{3 H^{2}}$ for matter and $\Omega_{\Lambda}=\frac{\Lambda c^{2}}{3 H^{2}}$ for cosmological term. Note that the present time values for the abundance components are $\Omega_{\Lambda 0}=0.7$ and $\Omega_{m 0}=0.3$ and the Hubble parameter is taken to be $H_{o}=$ $65 \mathrm{~km} / \mathrm{S} / M p c$ and the age of the universe is $T_{0}=\frac{2}{3} H_{0}^{-1}$.

Here we have assumed that $\Lambda \propto a^{-2 r}$ and $c \propto a^{-r}$ without keeping any constant and put into equation (8) to obtain the scale factor as a function of the cosmic time and the acceleration of the universe for the model of variable speed of light. The first instant is the case where $r=0$, and equation $(8)$ reduced to

$$
\frac{\ddot{a}}{a}+\frac{1}{2}\left(\frac{\dot{a}}{a}\right)^{2}-1=0
$$

The solution of the above equation is given by

$$
e^{\sqrt{6} t}=\frac{2 a(t)^{\frac{3}{2}} \sqrt{6} e^{\frac{\sqrt{6}}{2}} t}{3}
$$

And we obtain the scale factor to be given as

$$
a(t)=\left(\frac{\sqrt{6} e^{\sqrt{6} t}}{4 e^{\frac{\sqrt{6}}{2} t}}\right)^{\frac{2}{3}}
$$

The rate of expansion of the universe is shown in figure 1 below. After the Big Bang scenario, the universe has been in continuous exponential expansion as seen in equation (11).

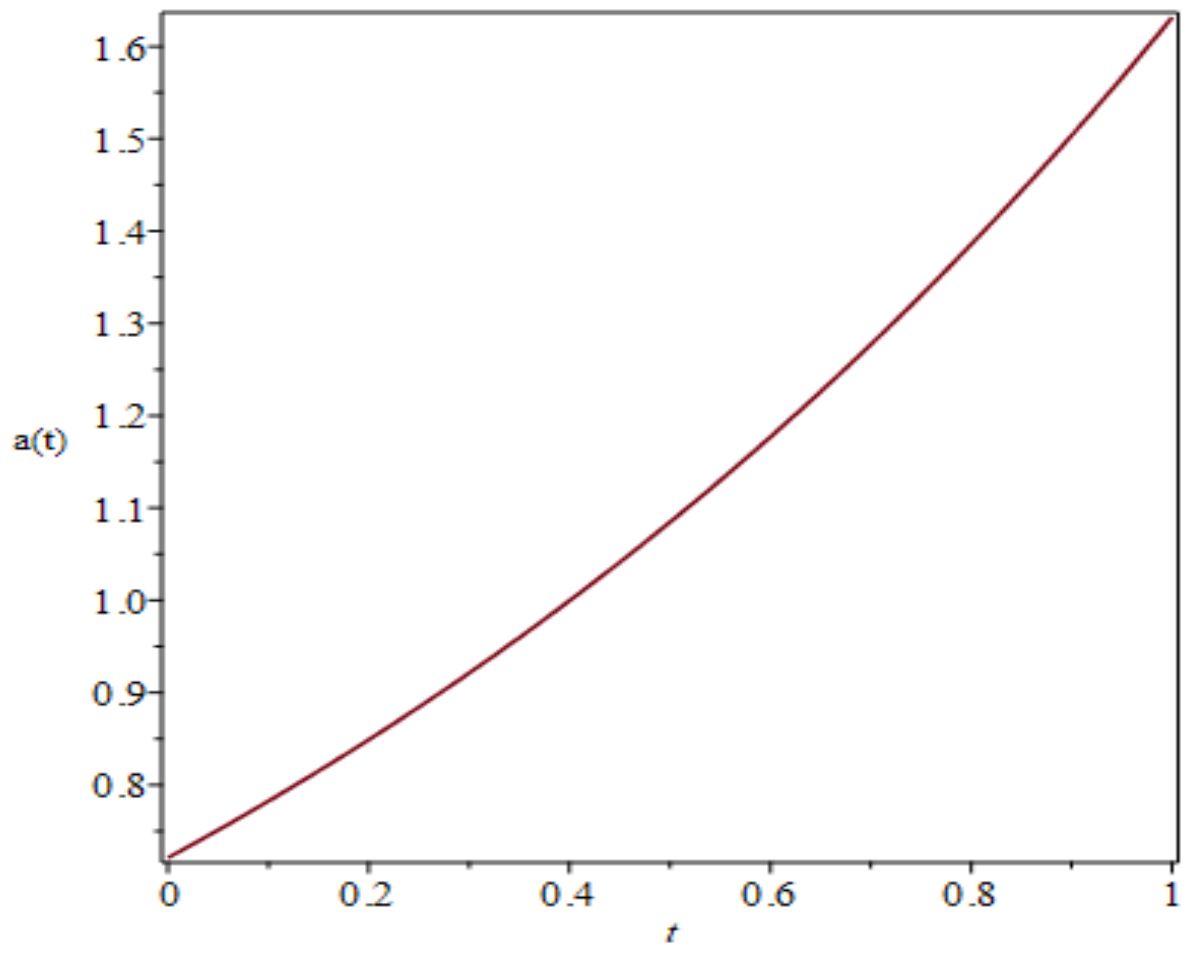

Figure 1. Variation of the Scale Factor with Cosmic Time for $r=0$ 
To check the acceleration of the varying speed of light model, we resolve to obtaining numerical solution of (8). The figure below show the cases in when $r=0.2$ and 1.0

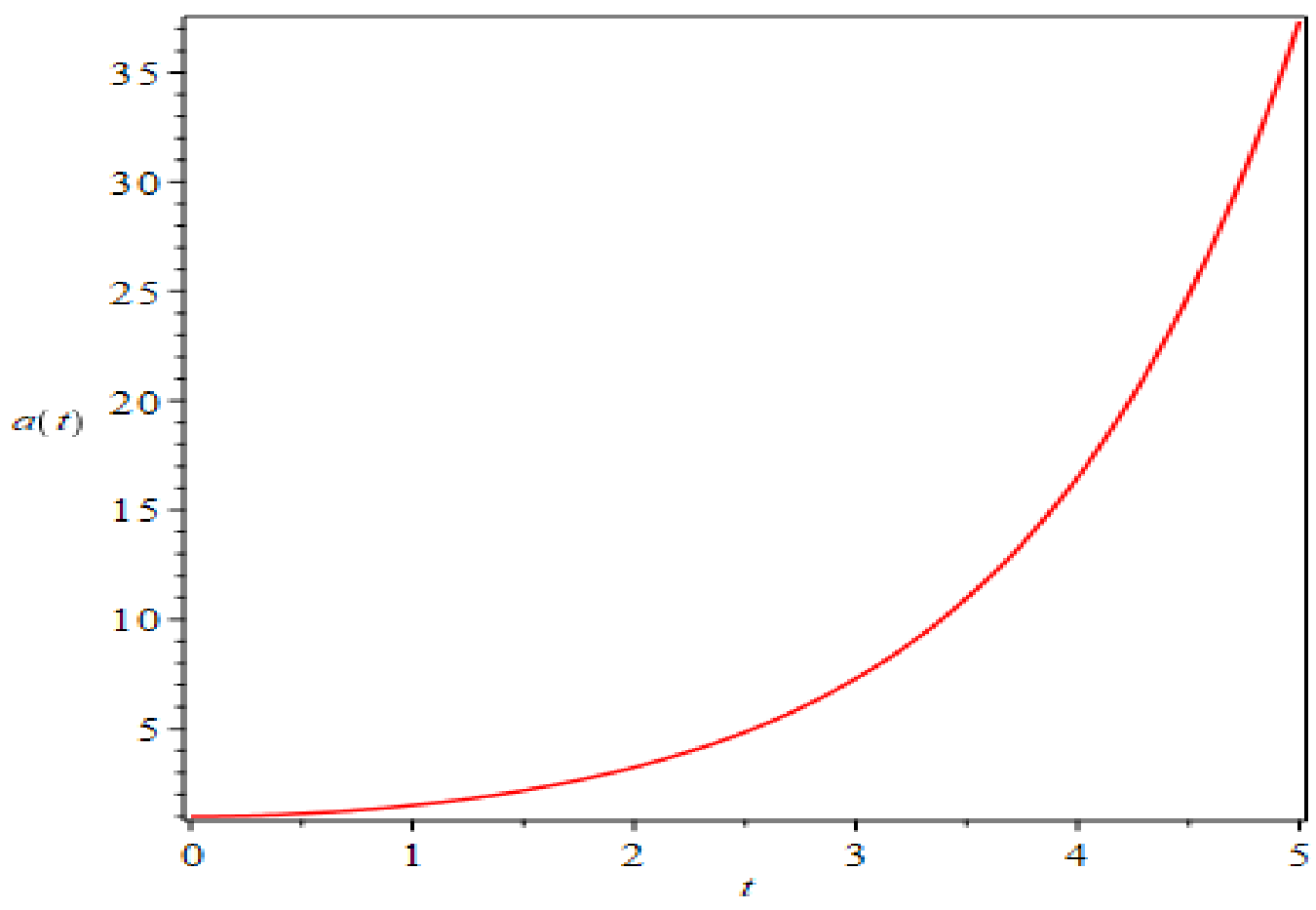

Figure 2. The Expansion of Universe for $\mathrm{r}=0.2$

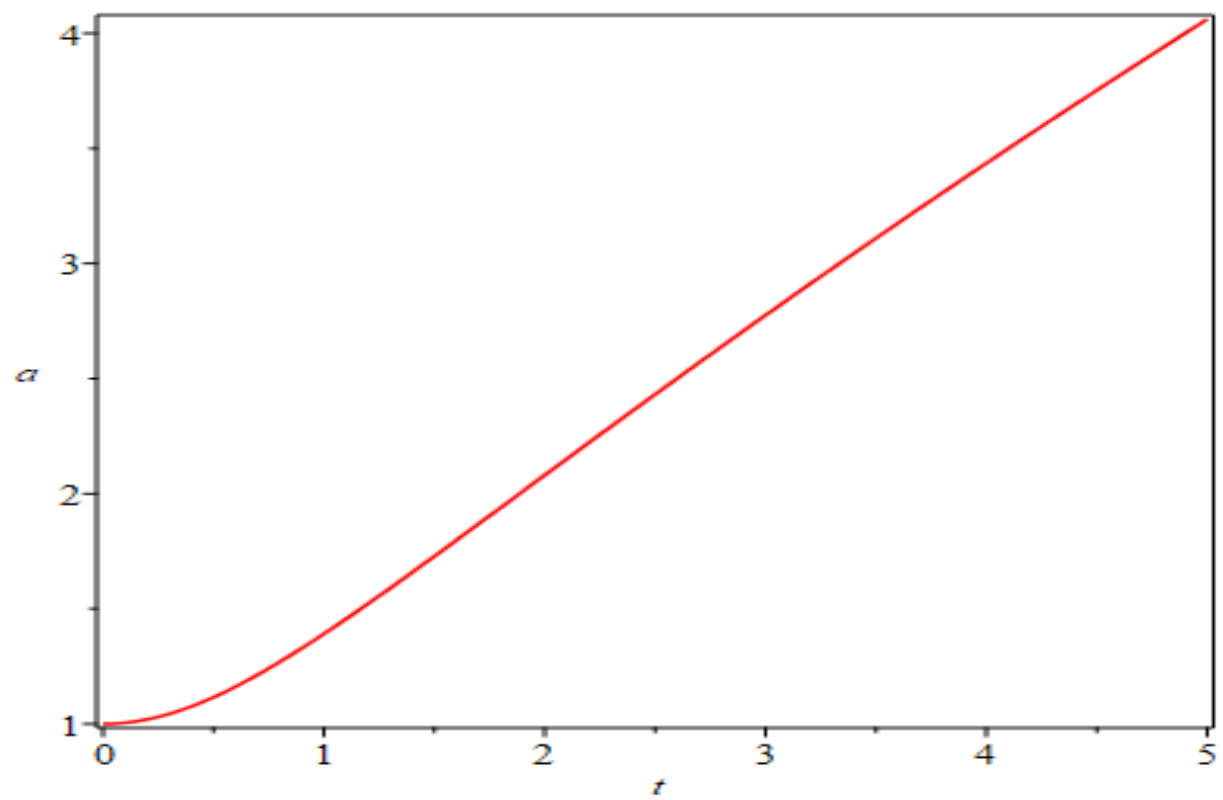

Figure 3. The Expansion of Universe for $r=1.0$ 
The solution is singular with the evolution of the universe and as $r \rightarrow 1$, the solution becomes nonsingular and inflationary model, implying $\ddot{a} \geq 0$. Hence, the model presents an accelerating one which is a good candidate for studying dark energy. The universe evolves from a singularity with $r=0$ and undergoes rapid exponential expansion. This is characterized by what is today known as cosmic inflation. As $r$ increases, the exponential expansion slows down as shown in figure 2 .

\section{Variation of the Speed of Light as a Function of the Scale Factor of the Universe}

If $d_{0}$ is the separation between two distant galaxies, then their separation at any given time is given by [14];

$$
d=a(t) d_{0}
$$

At the present time $t_{0}$, the scale factor is $a\left(t_{0}\right)=1$. Hence, at earlier time, $\mathrm{a}(\mathrm{t})<1$, at later time, $\mathrm{a}(\mathrm{t})>1$ and at big bang, $\mathrm{a}(\mathrm{t})=0$. The rate of change of distance gives the relative velocity $v$, of a pair of galaxies as $v=\dot{d}$. From equation (12) we obtain

$$
v=\dot{a}(t) d_{0}
$$

Assuming that in the early universe, particles move at the speed of light, from equation, we define the ansatz of

$$
c \propto \dot{a}(t)
$$

Using the relation of the variation of the cosmological constant with the scale factor of the universe in [11] given by:

$$
\Lambda=\frac{a_{0}}{a(t)^{2}}
$$

Appling the above equations into equation (8) we obtained:

$$
\ddot{a}+\left(\frac{3 \omega-2}{2}-\frac{\omega A}{2}\right) \frac{\dot{a}^{2}}{a}=0
$$

Where $A=a_{0} c_{0}^{2}$ is a constant.

Solving equation (16), we obtain a solution of the form

$$
a(t)=C_{1}\left(A \omega t-3 \omega t+2 C_{2}\right)^{\frac{-2}{\omega(A-3)}}
$$

Where $C_{1}$ and $C_{2}$ are real constants and simplifying with appropriate boundary conditions yields

$$
a(t)=a_{0}\left[\left(\frac{3-A}{2}\right) \omega t H_{0}\right]^{\frac{2}{\omega(3-A)}}
$$

Equation (18) gives the scale factor as a function of cosmic time for variable speed of light which depends on the equation of state parameter. The rate of expansion of the universe is affected its content. Indeed the speed of light would not have been constant in a multiple universe, whose content changes over different cosmic epoch.

Taking $a=a_{0}$ and $t=t_{0}$, we use equation (18) to obtain the present age of the universe to be

$$
t_{0}=\frac{2}{(3-A) \omega} H_{o}{ }^{-1}
$$

The present age of the universe is estimated by $H_{o}{ }^{-1}$ (where $H_{0} \approx 72 \mathrm{~km} / \mathrm{s} / \mathrm{mpc} \equiv 2.3 \times$ $10^{-18} \mathrm{~s}$ ). The age of the universe is estimated from the Hubble time to be about 13.79 billion years which agrees with observations from cosmic microwave background radiation. The question is, will the Hubble constant change in the nearest future as the universe advances in age? The simple answer remains no. There is need for a modified equation for estimating the age 
of the universe. For a single component universe, [15] expressed the current age of the universe as $t_{0}=\frac{2}{3(1+\omega)} H_{o}{ }^{-1}$. Equation (19) gives us a good approximation to estimating the current age of the universe with a good choice of the constant $A \neq 3$. Since $\mathrm{A}$ is either a negative or positive constant constant, it can be fixed to suit an component of any described universe.

The Hubble parameter $H=\frac{\dot{a}}{a}$ is given by

$$
H=\frac{1}{\left(\frac{3-A}{2}\right) \omega t}
$$

The deceleration parameter $q(t)$ in terms of the Hubble parameter is given as

$$
q(t)=-1-\frac{\dot{H}}{H^{2}}=-1+\frac{\omega}{2}(2-A)
$$

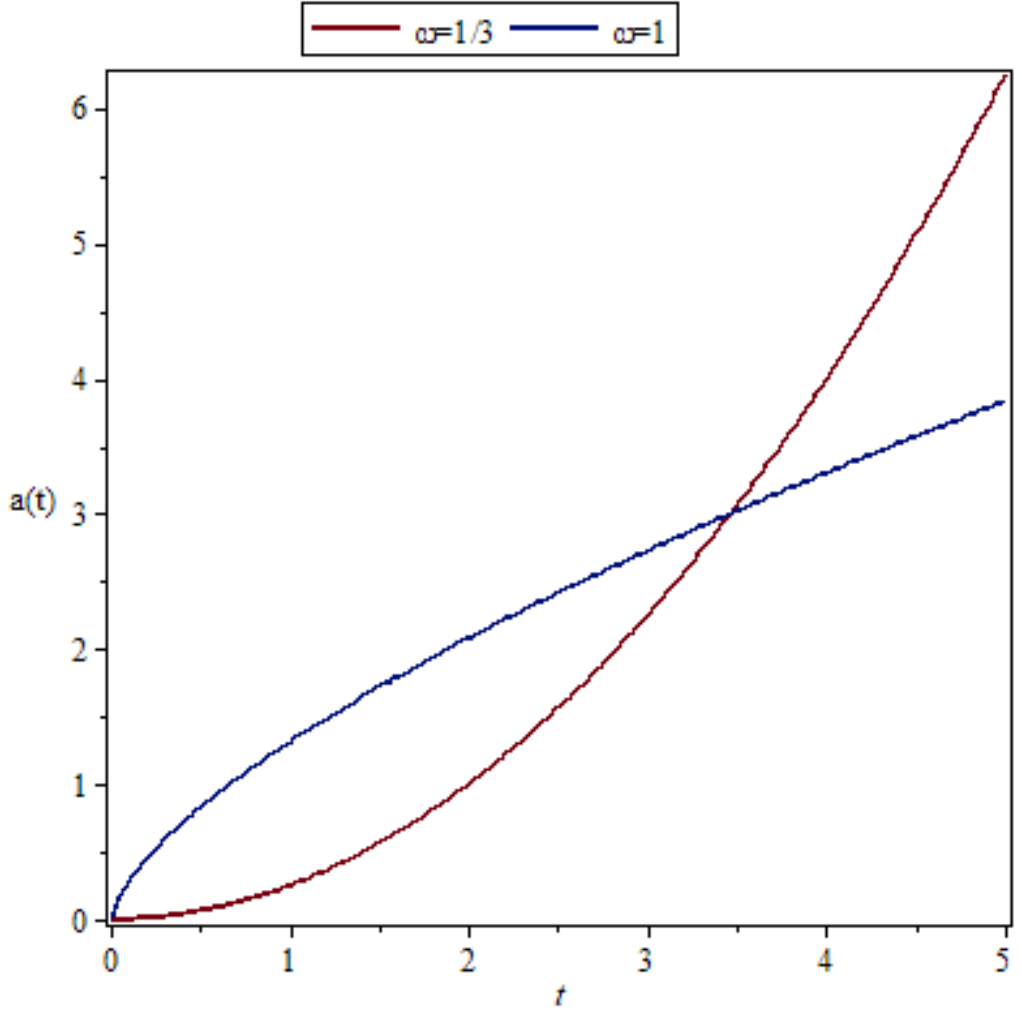

Figure 4. Variation of scale factor with cosmic time for $\mathrm{c} \propto \mathrm{a}(\mathrm{t})$ 


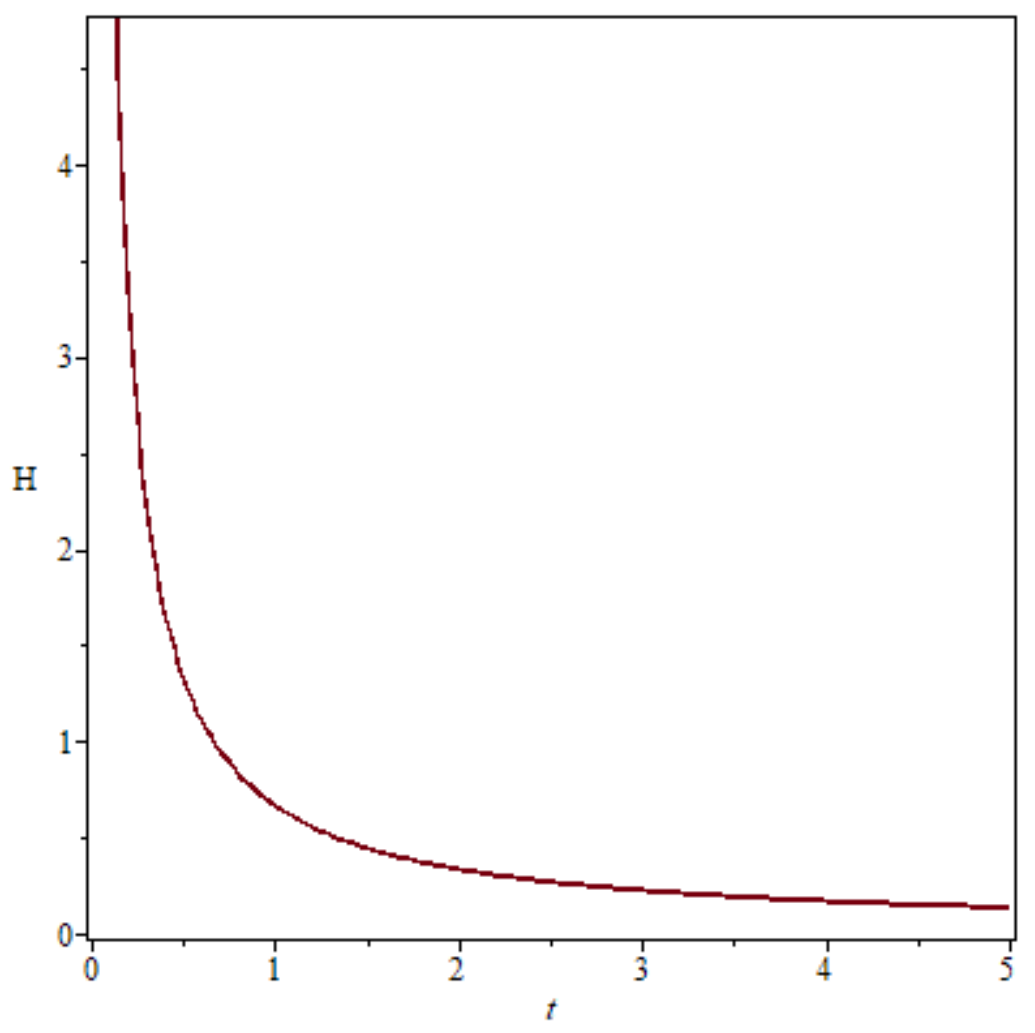

Figure 5. Variation of Hubble Parameter with cosmic time for $\mathrm{c} \propto \dot{a}(t)$

\section{CONCLUSION}

Cosmic models in which physical constants of nature are not constant but vary with time have been of interest in recent years. Indeed, the speed of light in our universe which has gone through various phase transition due to various content might not have been a constant especially at the early stage. We obtained the scale factor equation (18) which depends on the content of the universe given by the equation of state parameter $\omega$. From figure 4 , we observed that the universe was in its deceleration phase when $\omega=1$ and enters to the present acceleration when $\omega=\frac{1}{3}$. From figure 5, it is observed that the Hubble parameter diverges at the beginning and end of the universe. Such a universe starts with a positive deceleration parameter indicating deceleration expansion and transits to a negative deceleration parameter indicating accelerating expansion phase. Finally, with the aid of appropriate constant A, the model gives a modified way for estimating the age of the universe.

\section{REFERENCES}

1 Camara, C. S., Nascimento, R. B., Carvalho, J. C., \& De Garcia Maia, M. R. 2007. Solutions of cosmological models with varying speed of light. Int. J. Mod. Phys. D, Vol. 16, No. 02n03, pp. 433-437.

2 Singha, A. K., \& Debnath, U. 2007. Varying speed of light, modified Chaplygin gas and accelerating universe. Int. J. Mod. Phys. D, Vol. 16, No. 01, pp. 117-122. 
3 Perlmutter, S., Aldering, G., Goldhaber, G., Knop, R. A., Nugent, P., Castro, P. G., ... \& Supernova Cosmology Project. 1999. Measurements of $\Omega$ and $\Lambda$ from 42 high-redshift supernovae. Astrophys. J., Vol. 517, No. 2, pp. 565.

4 Jamil, M., \& Debnath, U. 2011. FRW cosmology with variable G and $\Lambda$. Int. J. Theor. Phys., Vol. 50, No. 5, pp. 1602-1613.

5 Gleiser, M. 1998. Phase transitions in the Universe. Contemp. Phys., Vol. 39, No. 4, pp. 239-253.

6 Hoyle, F., \& Narlikar, J. V. 1964. A new theory of gravitation. Proc. R. Soc. London. Ser. A. Math. Phys. Sci., Vol. 282, No. 1389, pp. 191-207.

7 Carroll, S. M., Press, W. H., \& Turner, E. L. 1992. The cosmological constant. Annu. Rev. Astron. Astrophys., Vol. 30, No. 1, pp. 499-542.

8 Berman, M. S., \& Som, M. M. 1990. Whitrow-Randall's relation and Brans-Dicke cosmology," Gen. Relativ. Gravit., Vol. 22, No. 6, pp. 625-629.

9 Ambrosi, G., An, Q., Asfandiyarov, R., Azzarello, P., Bernardini, P., Bertucci, B., ... \& Zhang, Z. Y. 2017. Direct detection of a break in the teraelectronvolt cosmic-ray spectrum of electrons and positrons. Nature, Vol. 552, No. 7683, pp. 63.

10 Al-Rawaf, A. S., \& Taha, M. O. 1996. Cosmology of general relativity without energymomentum conservation. Gen. Relativ. Gravit., Vol. 28, No. 8, pp. 935-952.

11 Singh, K. P. 2011. On Robertson-Walker universe model with variable cosmological term and gravitational constant in cosmological relativity theory. Turkish J. Phys., Vol. 34, No. 3, pp. 173-180.

12 Melia, F. 2016. Physical basis for the symmetries in the Friedmann-Robertson-Walker metric. Front. Phys., Vol. 11, No. 4, pp. 119801.

13 Moffat, J. W. 2005. Variable speed of light cosmology and bimetric gravity: An Alternative to standard inflation. Int. J. Mod. Phys. A, Vol. 20, No. 06, pp. 1155-1162.

14 Perlov, D., \& Vilenkin, A. 2017. Cosmology for the Curious. Springer.

15 Ryden, B. 2017. Introduction to cosmology. Cambridge University Press. 